\title{
PERFORMANCE ANALYSIS OF VOIP OVER WIRED AND WIRELESS NETWORKS : NETWORK IMPLEMENTATION IN ADEN UNIVERSITY
}

\author{
Mohsen Hussein Mohammed ${ }^{1}$, Wafa'a Nasser Abdullah ${ }^{2}$ \\ ${ }^{1}$ Associate Professor, Department of Information Technology, Engineering College, Aden, Yemen \\ ${ }^{2}$ Research Scholar, Department of Information Technology, Engineering College, Aden University, Aden, Yemen
}

\begin{abstract}
In this Paper, the objective of simulation models is presented to investigate the performance of VoIP codecs over WiMAX and FDDI networks that specially design for Aden University. To assure if the University IP network is prepared and adequate for this new type of traffic before adding any new components, Aden University IP network will be simulated by using OPNET simulation software then the new VoIP service will be added to the University networks. Different parameters that represent the QoS like end to end delay, jitter, traffic sends and traffic received, MOS are calculated and analyzed in both network scenarios.
\end{abstract}

Keywords: VoIP, Codecs, QoS, WiMAX, FDDI.

\section{INTRODUCTION}

Through the last few years, there was a progression trend into real-time voice communication using Internet protocol (IP).This progress caused by the merger of voice and data traffic (telecommunication convergence) over the existing network infrastructure, improved network management and lower cost offered by technology. VoIP (Voice over Internet Protocol) is a communication technology that supports delivery of voice over the Internet or other packet switched networks rather than the Traditional Public Switched Telephone Network (PSTN) [1]. The wired Internet Protocol (IP) networks given higher VoIP services as compared with the wireless IP network as wireless networks and they have their own characteristics and weakness [2]. The unsolved problems triggered via the wireless network in this place still need a few dedicated work spotlighting VoIP calls [3].

In this project, the OPNET simulator is used to implement the proposed VoIP network. We study all the potential parameters that can decline the quality of VoIP over two types of networks as FDDI and Wireless MAN (WiMAX) designed for Aden University. The study is depended on the choice of an appropriate voice compression and decompression (CODEC) schemes depending on the Quality of Service (QoS) of VoIP in variant networks. Different parameters that indicate the QoS like end-to-end delay, jitter throughput, and MOS, are calculated and analyzed. The comparisons were carried out between different codecs (G.711, G.729A, and G.723.1) which are the most appropriate to improve QoS for VoIP.

The whole parts of this paper are organized as follows. Some related works are mentioned in Part 2. Part 3 concerns with VoIP codec schemes. The model design and configuration networks are described in Part 4. Part 5 presents simulation results and analysis. Finally, Part6 concludes the paper.

\section{LITERATURE REVIEW}

Many authors have worked on diverse Quality of Service (QoS) parameters using unique service classes in different network types. A study was performed on various quality parameters influencing on the VOIP service performance. The study shows that these parameters of QoS are required to increase VoIP performance. Gihad Yousif Gafar [4], indicates that the simulation results have a significant effect on the performance of VoIP in WiMAX networks, which refer that better choice of voice codec's and statistical distribution and performance of selected parameters will be done using the network simulator OPNET Modeler. In Takahashi et al [5], the authors attempt to identify some of the network performance parameters that Service Providers will consciousness directly to develop a VoIP over WiMAX communication tool. That serves as a voice communication broadband alternative technology to old circuit-switched voice communication. Abdul-Bary Raouf Suleiman et al. [6], present a simulation for SIP-Based based VoIP in Mosul college community. Voice telephony is taken into consideration as a real-time service carrier and time sensitive service. The performance of network and voice quality examine after including the VoIP for distinct codec types. The results are presented that Mosul University IP network is successful to enforce the VoIP. Hussein et al, [7], linked a comparison between different queue algorithms. The authors found that Priority queue and Weight Fair Queuing algorithms are the most appropriate to improve QoS for VoIP. R. Nandhini et al. [8], represent that the objective of the broadband wireless technologies is to assure the end-to-end Quality of Service for the classes of service. The proposed algorithm describes in detail, and evaluates 
with real-time video traffic and one VOIP codec, by a series of simulations involved in this interesting location of studies. In [9] Ali M.Alsahlany, evaluated the VoIP performance based wireless LAN/WAN network with taking into account different voice encoding schemes. Different parameters that refer the QoS like jitter, end-toend delay, traffic sends, traffic receives and MOS measured and analyzed in Wireless LAN /WAN scenarios. However, all the previous studies are designed in the literature based on either wired or wireless network or both. Most of them designed for LAN or WAN networks. Therefore, it is essential to do a research on both wired and wireless MAN networks from different aspects. This paper examines VoIP performance of campus; MAN network, containing both wired and wireless network components. The results of this paper can help organizations make decisions for adopting VoIP system and expansion plans for VoIP services. A number of studies have published where the performance of several codecs evaluate and compare under different conditions such as the performance of codec on a Local Area Network (LAN) and wide area network (WAN) with different characteristics such as a number of users and different networks.

\section{VOIP CODECS}

Voice Codecs are used on the client side to convert the analog voice signal to digital signal and vice versa. There are various codecs types depended on the selected data rate, sampling rate, and implemented a compression algorithm listed in Table.1. In order to locate the bandwidth requirements of VoIP connections, we will begin by presenting common VoIP Codecs and their associated characteristics[10].

G.711 is currently used in a wide domain of applications. It employs a logarithmic compression that compresses each 16-bit sample to 8-bits. As a result, it digitizes voice into 64 kbps, which consider the highest bit-rate among the codecs.
It performs best in local networks where we hold lots of available bandwidths[10],[11].

G.729 is an authorized codec designed to deliver good call quality without exhaustion of high bandwidth. It builds based, on the Conjugate-Structure Algebraic-Code-Excited Linear Prediction (CS-ACELP) algorithm, with a bit rate of $8 \mathrm{kbps}[11]$.

G.723 is also an authorized codec. It is designed for calls over modem links with data rates of 28.8 and $33 \mathrm{kbps}$. It operates at 6.3 and $5.3 \mathrm{Kbps}$. Although this standard decreases bandwidth exhaustion, the voice is much poorer than with G.729 and is not very common for VoIP [11].

Table -1: VoIP Codec Characteristics

\begin{tabular}{|c|c|c|c|c|}
\hline CODEC & Coding Algo & $\begin{array}{c}\text { Samplin } \\
\text { g rate }\end{array}$ & $\begin{array}{c}\text { Packet } \\
\text { Per } \\
\text { Second }\end{array}$ & $\begin{array}{c}\text { IP } \\
\text { Packet } \\
\text { Size }\end{array}$ \\
\hline G.711 & PCM & $64 \mathrm{kbps}$ & 100 & 120 \\
\hline G.723.1 & ACELP & $5.3 \mathrm{kbps}$ & 33 & 60 \\
\hline G.729A & CS-ACELP & $8 \mathrm{kbps}$ & 100 & 50 \\
\hline
\end{tabular}

\section{NETWORK MODEL DESCRIPTION}

Our simulation approach uses OPNET simulator for network modeling. OPNET simulator is an authoritative communication system simulator developed by OPNET technologies. Two simulation models were to determine the best audio encoding schemes of utilizing VoIP over both WiMAX and FDDI networks. Both networks follow a different structure and the same topology. The basic difference in all the scenarios was in the configuration of the corresponding application and the profiles. Each scenario is implemented with the codec G.711, G.723, and G.729. Various comparisons are conducted to find the values of various parameters. The simulation models for the networks under study are illustrated in Figure 1 (a) and Figure 1 (b).

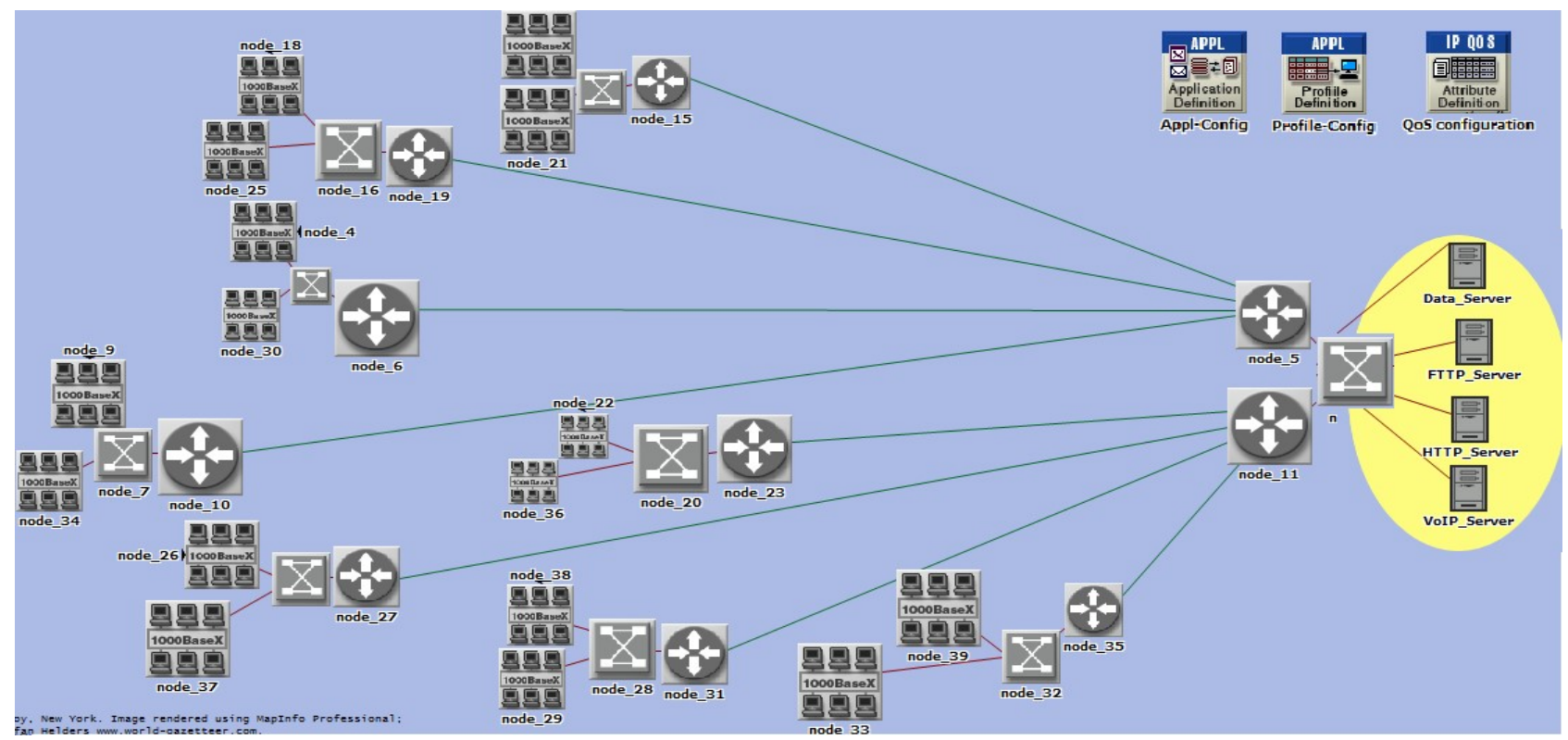

Fig -1(a): FDDI Network 


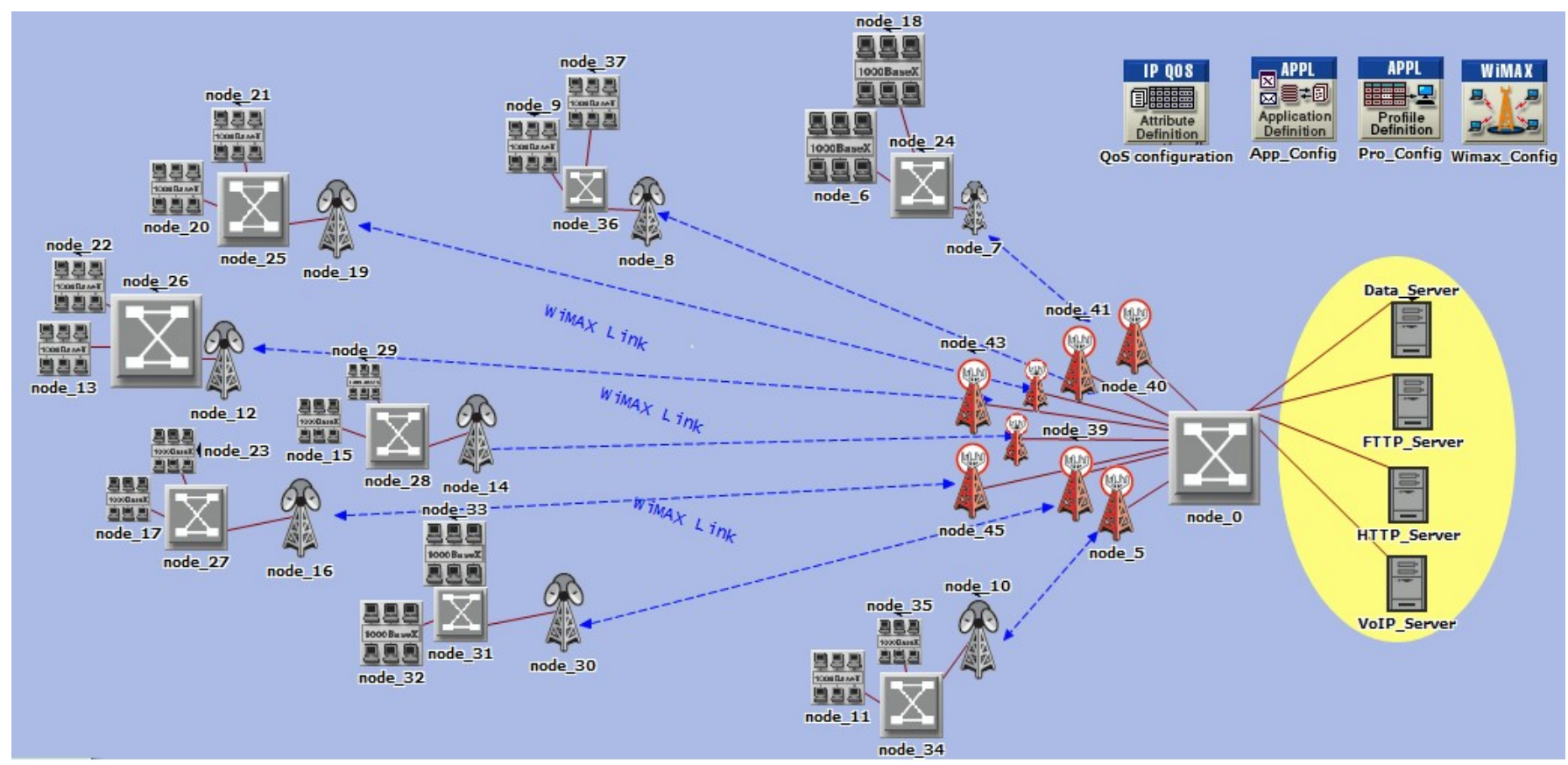

Fig -1(b): WiMAX Network

The Application Config, Profile Config, Mobility Config and QoS Attribute Config are included in the models. The experimental network models consist of Wired and Wireless network setup in the University campus. The wired model consists of two main routers, which are connected to eight different parts of campus by fiber optic links and each zone has an equal number of VoIP users. On the other side, Wireless network is similar to the wired network, where LANs in all the eight zones are the same, the connection between the center and each zone is replaced by wireless routers on the other hand. In a wireless network, we implemented SIP proxy server (session initiation protocol) to work together with FTP (file transfer protocol) and HTTP (Hyper Transfer Text protocol) as VoIP server.

\subsection{Basic Parameter}

Wireless MAN environment uses 1 Gbps Ethernet cable for all LAN networks. The OPNET model name for servers is 'ethernet_server'. The wireless MAN environment contains 1 wireless Router and 2 fixed workstations. All these nodes use VoIP services. There are a total of 16 workstations in each Wireless MAN environment. The router Opnet model names are 'WiMAX_ss_ethernet_slip_WLAN_router_adv' as WiMAX subscriber station routers and 'WiMAX_bs_ethernet4_slip4_router' as WiMAX base station routers. The fixed VoIP workstation model name is '1000BaseX_LAN'. The workstations are generating traffic across the LANs and the Wireless MAN environment to simulate a campus office environment.

Different types of connection connect all University buildings. With Wired network, fiber optic cables distribute around the University and connecting all campus buildings with the router Opnet model 'ethernet_fddi_gtwy_base'. Opnet modeler 14.0 is used as simulation software for the university campus network [12]. All parameters used in the wired and wireless MAN environment show in Table 2.
Table -2: Parameters Used In Simulation

\begin{tabular}{|c|c|}
\hline Parameter & Value \\
\hline Workstation & 1000BaseX_LAN \\
\hline Server & ethernet_server \\
\hline Switch & eth16_ethch16_fddi16_tr16_switch \\
\hline Router & ethernet_fddi_gtwy_base \\
\hline $\begin{array}{c}\text { WiMAX_bs } \\
\text { router }\end{array}$ & wimax_bs_ethernet_slip_wlan_router_adv' \\
\hline $\begin{array}{c}\text { WiMAX_ss } \\
\text { router }\end{array}$ & wimax_ss_ethernet_slip_wlan_router_adv' \\
\hline Links & - FDDI 100MB -1000BaseX duplex link \\
\hline
\end{tabular}

\subsection{Application, Profile and Configurations}

Application Definitions are used to create traffic generation on the network. There are four applications utilized in each scenario. Those are VoIP, FTP, web browsing, and database applications. The essential one is VoIP. Within the Voice category, IP Telephony was selected for configuration VoIP. Within the VoIP application, voice attributes were set-up as codec schemes G.711 for scenario 1; G.723.1 for scenario 2; and G.729A for scenario 3. G.729A runs at a bit rate of 8 Kbps, G.723.1 runs at a bit rate of 5.3/6.3 Kbps and G.711 runs at a bit rate of $64 \mathrm{Kbps}$. For the QoS scenarios, the VoIP table type of service (ToS) is changed into six to offer voice priority. Different parameters that have been set for this venture were internet browsing and file transfer. For each of these applications stated, there has been a heavy usage parameter set. 
Profile Configuration describes the activity applications used by users through the duration. VoIP Profile was only used with VoIP applications that were the committed VoIP phones. All of the Profiles configured to run simultaneously to permit more than one application to be operated at the same time.

\section{SIMULATION RESULTS AND ANALYSIS}

The following figures are obtained by accumulating statistics by using the OPNET Modeler Simulation tool. All figures show a comparative picture of the 3 scenarios. All of the 3 scenarios in each network use a specific audio codec scheme consisting of three codecs G.711, G.723 and G.729. After running the simulation successfully, the final result suggests the impact of various codecs on different QoS parameters in a VoIP network. The following are the figures shown below display different QoS parameters like packet end to end delay, Voice jitter, Voice traffic sends (packet/Sec) and Voice traffic receives (packet/Sec), and MOS.

\section{End to End Delay}

The delay is the amount of time that takes a packet from one end of the network to the other. Unlike many data applications, voice can't afford high levels of latency or delay. Therefore, for any network to be more efficient, it must have less delay value. It is calculated in terms of sec or min. The Following graphs of delay shown in figure 3 (a) and 2 (b) are drawn between the simulation time and basic time, which is termed as the delay of the network from both graphs. G711 has less delay with compared to the others. Therefore, we can say that these networks are more efficient in this delay term in wired network. In figure 2(a) both G.711 and G.29 have the same delay value and this value is less than the other network, while a voice delay of the G.723 codec scheme becomes worse. In figure 2(b), G.711 has less delay value than the other network scenarios. From both graphs, G.711 is much better in WiMAX and G.711 and G.29 are much better in wired networks.

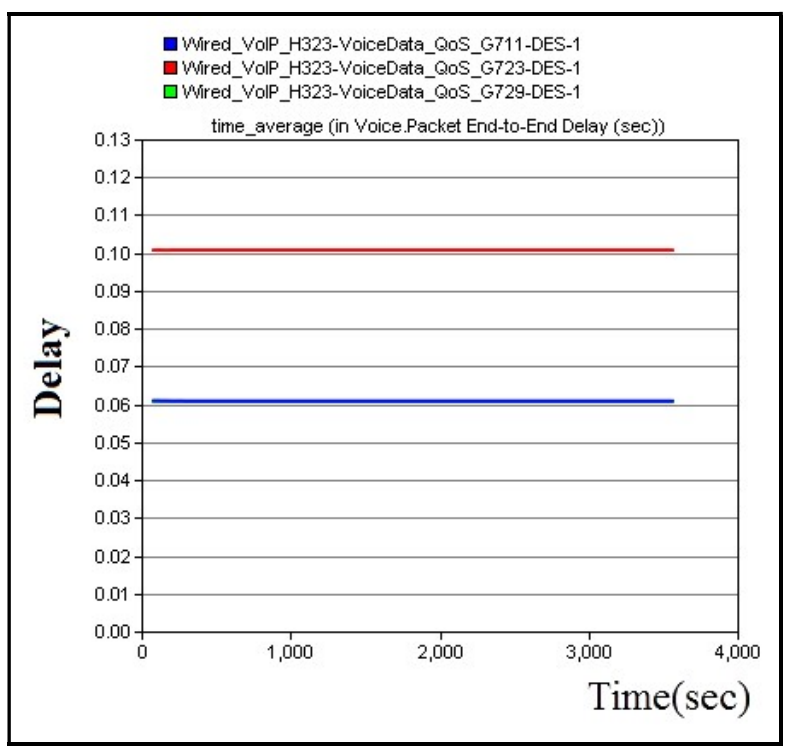

Fig -2 (a): FDDI (Voice ETE Delay)

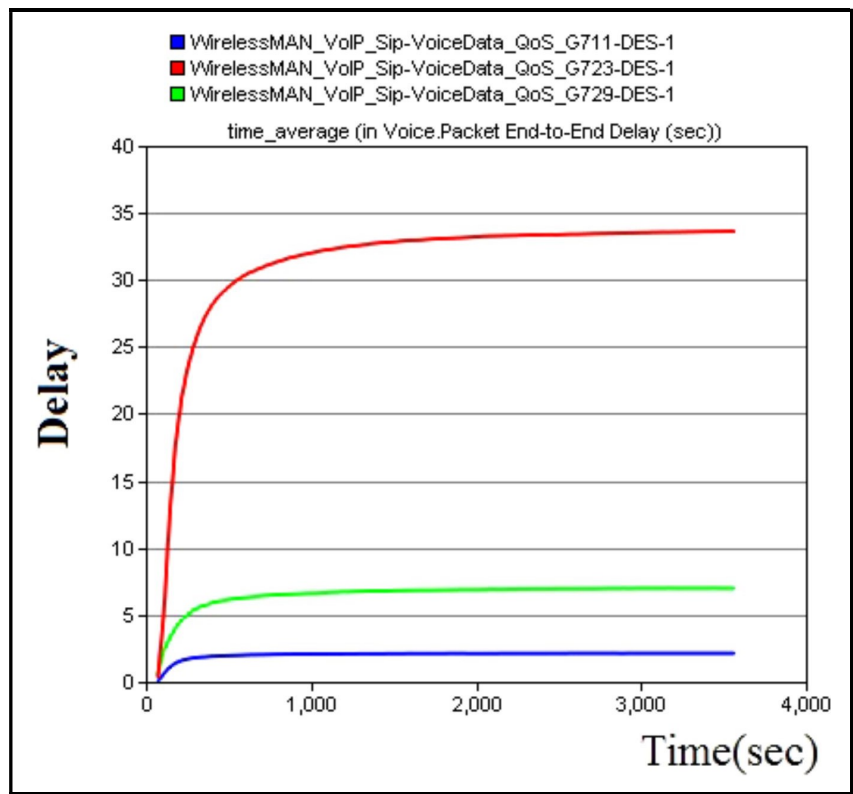

Fig -2(b): WiMAX (Voice ETE Delay)

\section{Jitter}

Jitter is the value, which represents the time taken by packets to reach the destination. This variation is caused due to route changes, timing drift or network congestion. It is calculated in terms of Sec or minute. Perceived voice quality is the best if the jitter is zero. These graphs are drawn between the simulation time and basic time, which is termed as jitter of the network. In figure 3(a) graph, the jitter of G.711, G.723, and G.729 decrease and become very close to zero. In figure 3(b) graph, G.711 has the best jitter value and this value less than other network scenarios. Therefore, these networks are better in this jitter term. From both graphs, G.711 is much better.

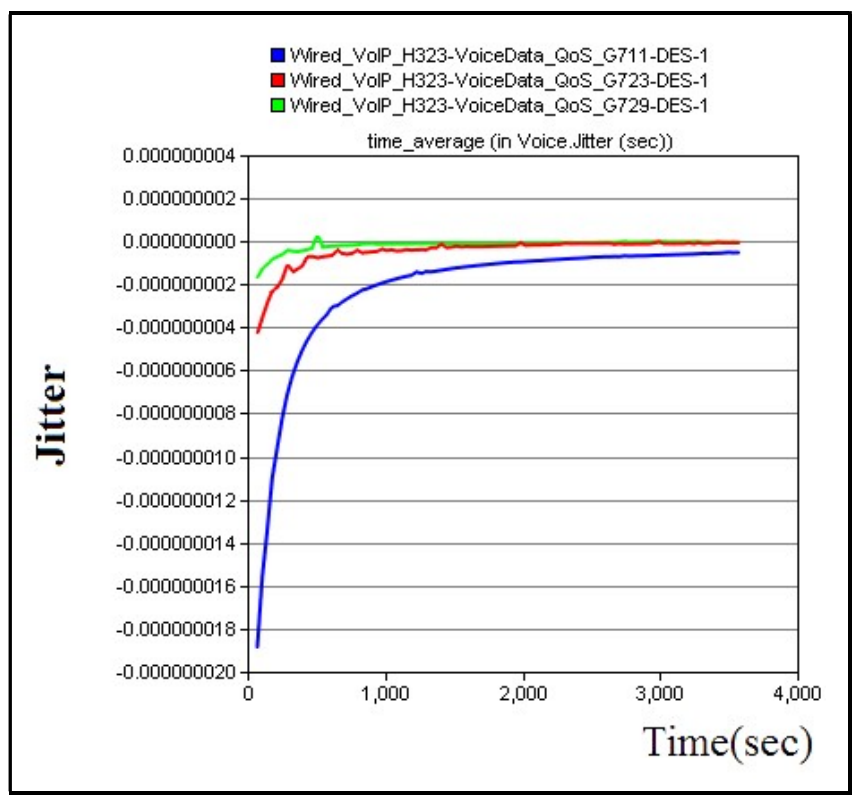

Fig -3 (a): FDDI: Voice Jitter 


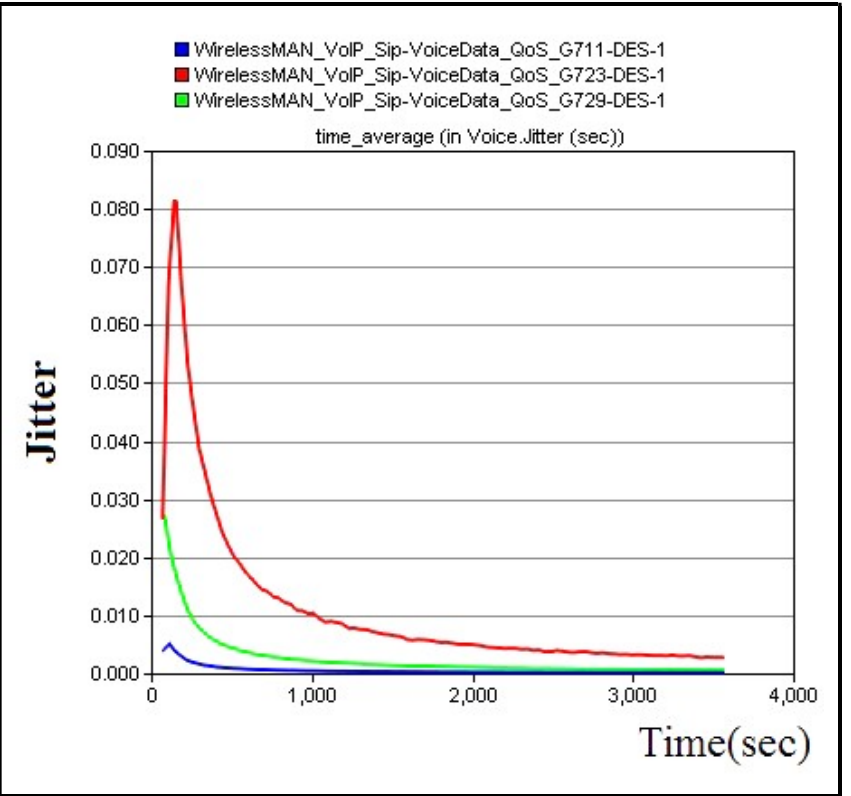

Fig. 3(b): WiMAX (Voice Jitter)

\section{Throughput}

Figure 4(a) and 4(b) are the graphs of traffic throughput in both figures; two traffics must be the same for any network to be more efficient. In Figure 4(a) network scenarios indicate that the G.729 scenario is the best in traffic send and receive in comparison with other scenarios. Otherwise, Figure 4(b) scenarios, traffic received by the network with G.711 is more deviated from the traffic sent, whereas the G.729 network, the deviation is much less. This analysis indicates that the noise added in the G.729 scenario is less with compared to other scenarios. Therefore, we can say that this network is more efficient.

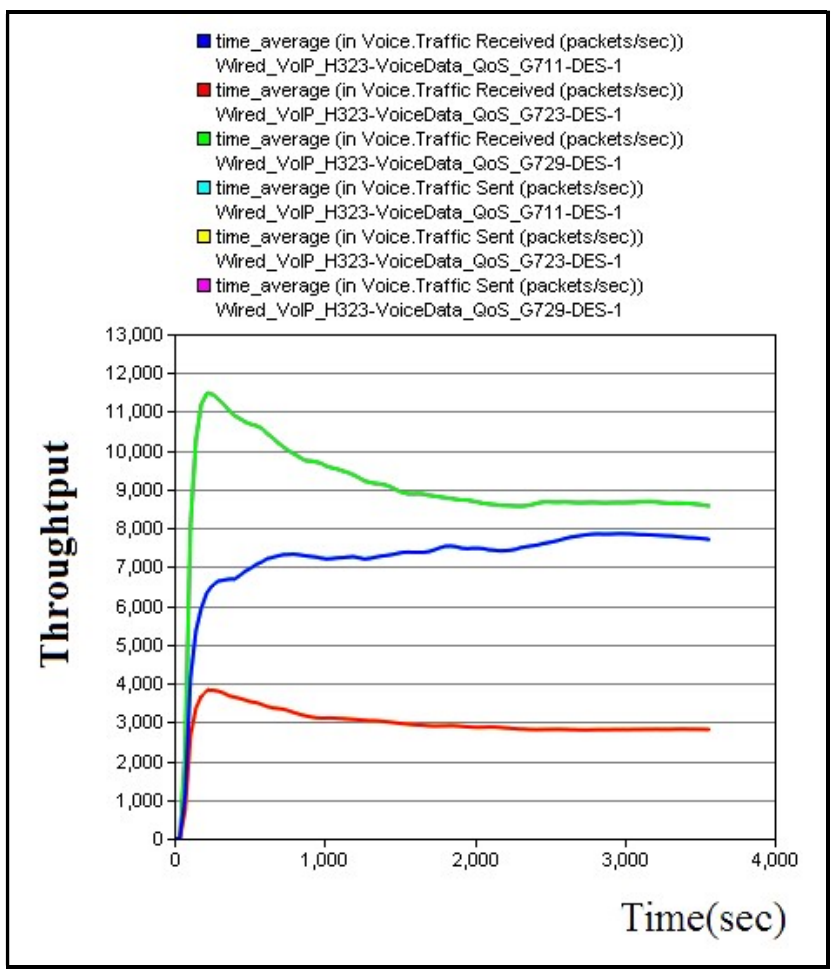

Fig -4(a): FDDI (Voice Throughput)

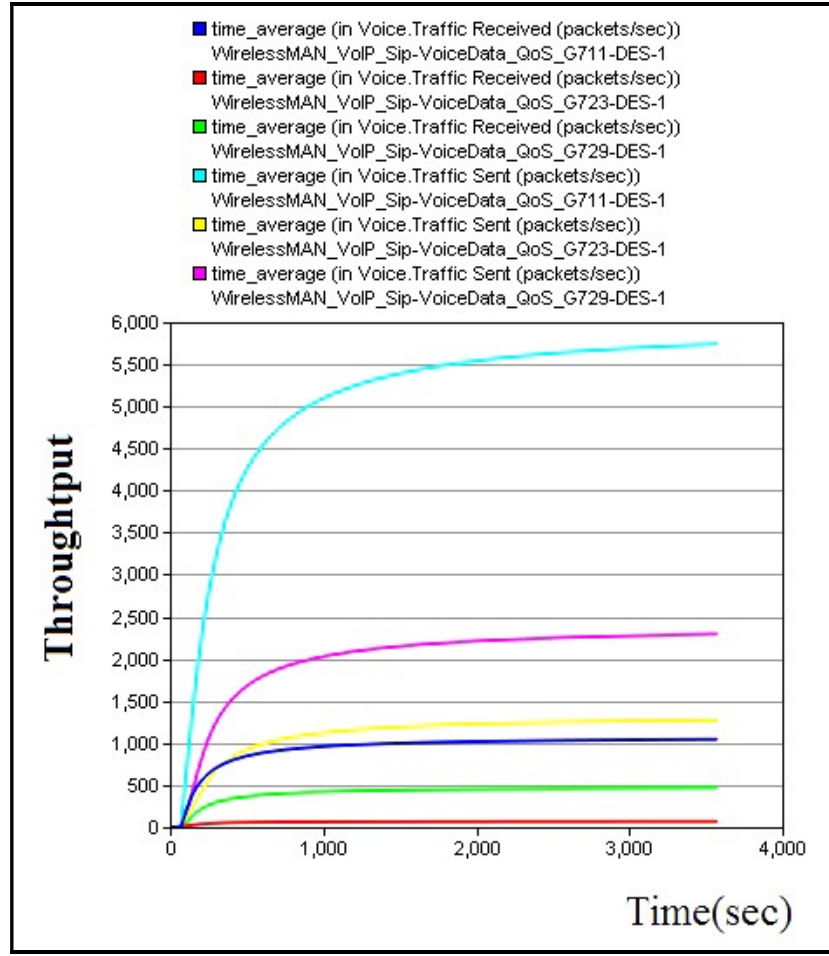

Fig -4(b): WiMAX (Voice Throughput)

\section{Mean Opinion Score (MOS)}

MOS (Mean opinion score) In the VoIP, MOS is a number between 1 and 5 used to describe the voice perception quality in communication systems, especially digital networks that transfer VoIP traffic. Therefore, Voice quality measures by the Mean Opinion Score (MOS). Figures 5(a) and 5(b) display the MOS factors of our networks for three Codec types. We observe that the MOS factors for Codecs G. 711 and G. 729 have acceptable MOS values 3.685 and 3.067 , respectively. On the other hand, the MOS values for G. 723 are 2.557 in FDDI network and 1 in WiMAX network, which indicates that the quality of service is poor and unacceptable if this codec is used.

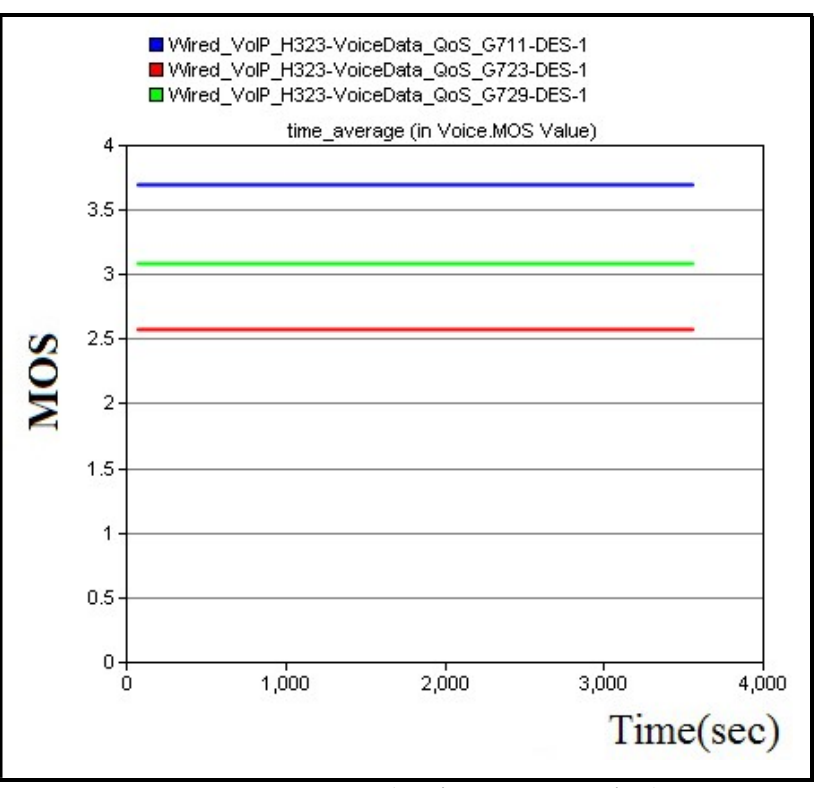

Fig -5(a): FDDI (Voice MOS Value) 


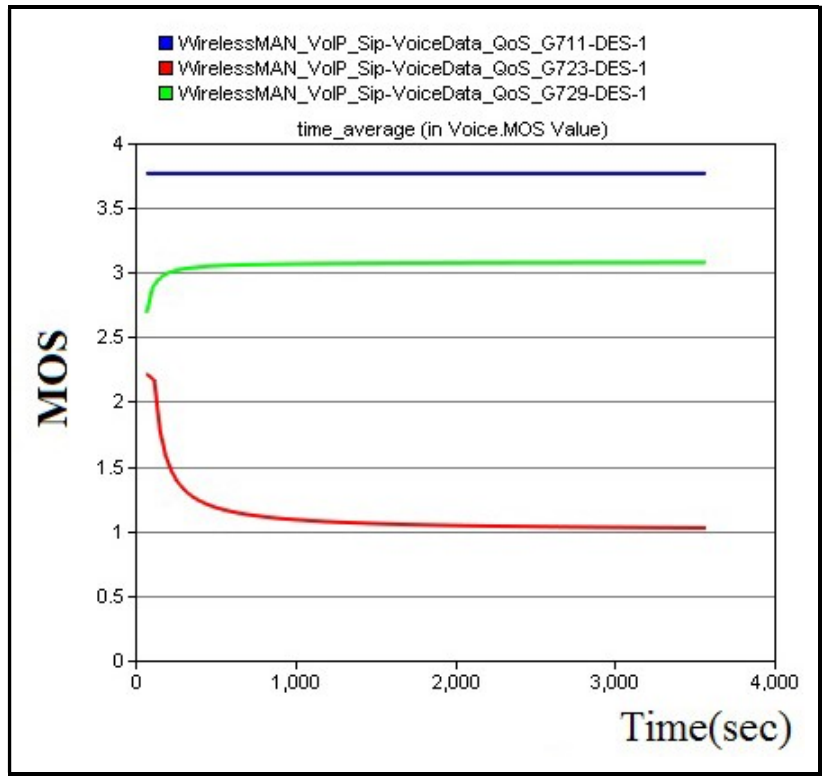

Fig -5(b): WiMAX (Voice MOS Value)

\section{CONCLUSIONS}

In this implementation, we evaluated the performance of VoIP codecs over both FDDI and WiMAX networks. Three voice codecs G.711, G.723, and G.729 simulated in order to determine the most appropriate voice codec for VoIP over FDDI and WiMAX network. The VoIP performance simulate via six simulation scenarios by using OPNET 14.0. Different parameters such as end-to-end delay, jitter, throughput, and MOS, are used to measure VoIP performance. The implementation results of this paper show that the VoIP services perform best under G.711 voice encoder scheme over WiMAX network. Otherwise, in FDDI network, VoIP services perform best under both G.711 and G.729 voice encoders schema. In addition, in FDDI network voice throughput is higher in codec G.729 as compared to the other two referenced codecs.

The future research can be done in advance wireless standard and equipment for WiMAX network by implementing the network in the latest edition of OPNET MODELLER 17. In addition, calculating the performance of codec includes the combination between more than one codec to achieve a VOIP application using different networks. FDDI and WiMAX networks may also use other VoIP codecs such as G. 722, G. 726, GSM, and G.728.

\section{REFERENCES}

[1]. S. Dhanalakshmi, and M. Sathiya, "Investigating The Performance Of VoIP Over Ethernet LAN In Campus Network" International Journal of Recent Scientific Research,Vol. 6, Issue, 6, pp.4389-4394, June, 2015.

[2]. A.Samukic,"Umts Universal Mobile Telecommunications System Development of Standards for the Third Generation“, 1998.

[3]. Malik Ahsan Ali, Imran Rashid, and Adnan Ahmed Khan, "Selec-ion of VoIP CODECs for Different Networks based on QoS Analysis" International Journal of Computer Applications (0975 - 8887) Volume 84 - No 5, December 2013.
[4]. Gihad Yousif Gafar, "Enhanced Quality of Service in Worldwide Interoperability for Microwave Access Networks", IOSR Journal of Electronics and Communication. Engineering (IOSR-JECE), Volume 9, Issue 5, Ver. V (Sep Oct. 2014), PP 72-78.

[5]. Elechi Onyekachi O.1, and Eze Elias C.1*, "Investigating the QoS of Voice over IP using WiMAX Access Networks in a Campus Network", Computer, Engineering and Intelligent Systems, ISSN 2222-1719 (Paper) ISSN 2222-2863 (Online), Vol.4, No.5, 2013.

[6]. Abdul-Bary Raouf Suleiman \& Abdul-Hameed Hameed "Simulation of SIP-Based VoIP for Mosul University Communication Network", International Journal of Computing and Digital Systems, Int. J. Com. Dig. Sys.2, No. 2, 89-94(2013).

[7]. Hussein, and et al, "The Effects of Different Queuing Algorithms within the Router on QoS VoIP application Using OPNET,'International Journal of Computer Networks \& Communications (IJCNC) Vol.5, No.1, Jan, pp. 117-124, 2013.

[8]. R.Nandhini, N. Devarajan, "Channel quality based cross-layer scheduling algorithm in Wimax networks" available online: http://www.lifesciencesite.com, Life Science Journal 2013; 10(2).

[9]. Ali M. Alsahlany, "Performance Analysis of VoIP Traffic Over integrating Wireless LAN and WAN Using Different Codecs", International Journal of Wireless \& Mobile Networks (IJWMN) Vol. 6, No. 3, June 2014.

[10]. Tarik Anouari and Abdelkrim Haqiq, "Performance Analysis of VoIP Traffic in WiMAX using various Service Classes", International Journal of Computer Applications (0975 - 8887), Volume 52- No.20, August 2012.

[11]. Gysberth Maurits Wattimena, "Analysis Performance VoIP Codecs over WiMAX Access Network “,International Journal of Advanced Research in Computer Science and Electronics Engineering Volume 1, Issue 7, September 2012 [12].OPNET,http.//www.telecomlab.oulu.fi/kurssit/521365 A_tietoliikennetekniikan_simuloInnit_ja_tyokalut/Opnet_es ittely_07.pdf, journal, Retrieved. 2013-12-05.

\section{BIOGRAPHIES}

Mohsen Hussein Mohammed associate professor, vice Dean of students Affairs in Faculty of Computer and Information Technology - Aden University- Yemen. He has received the $\mathrm{PhD}$ degree in Computer Systems, Complexes and Networks from the Faculty of Engineering Blagoevgrad University-Bulgaria by 2006 . He has published a number of researches in various journals and international conferences. His current research interests are in parallel computer architecture, Multicomputer platform, System area Networks. E-mail: mohsen_al_hedry@yahoo.com

Wafa'a Nasser Abdullah research scholar, she has worked as a lecturer in the Computer Department at Zanzibar Education College- Aden University since 2010 till now. She received the B.S of Engineering degree from Department of Information Technology - Faculty of Engineering- Aden University, Yemen, in 2009, Her current research interests are in VOIP over WiMAX network. E-mail:wafa7903@yahoo.com 